\title{
Invertebrates for vertebrate biodiversity monitoring: comparisons using three insect taxa as iDNA samplers
}

\author{
Aimee Massey ${ }^{1}$, Roberta Bronzoni ${ }^{2}$, David da Silva ${ }^{2}$, Jennifer Allen ${ }^{1}$, Patrick de Lazari ${ }^{3}$, \\ Manoel dos Santos Filho ${ }^{3}$, Gustavo Canale ${ }^{2}$, Christine Bernardo ${ }^{2}$, Carlos Peres ${ }^{4}$, and Taal \\ Levi $^{1}$
}

${ }^{1}$ Oregon State University

${ }^{2}$ Universidade Federal de Mato Grosso - Campus Sinop

${ }^{3}$ Universidade do Estado de Mato Grosso

${ }^{4}$ University of East Anglia

April 29, 2021

\begin{abstract}
Metabarcoding of environmental DNA (eDNA) is now widely used to build diversity profiles from DNA that has been shed by species into the environment. There is substantial interest in the expansion of eDNA approaches for improved detection of terrestrial vertebrates using invertebrate-derived DNA (iDNA) in which hematophagous, sarcophagous, and coprophagous invertebrates sample vertebrate blood, carrion, or feces. Here, we use metabarcoding and multiple iDNA samplers (carrion flies, sandflies, and mosquitos) to profile gamma and alpha diversity in a dry, tropical forest in the southern Amazon. Our main objectives were to (1) compare diversity found with iDNA to camera trapping, which is the conventional method of vertebrate diversity surveillance and (2) compare each of the iDNA samplers to assess the effectiveness, efficiency, and potential biases associated with each sampler. Carrion flies were the most effective sampler, despite the least amount of sampling effort and the fewest number of individuals captured for metabarcoding, in describing vertebrate biodiversity followed by sandflies. Camera traps had the highest median species richness at the site-level but showed strong bias towards carnivore and ungulate species and missed much of the diversity described by iDNA methods. Mosquitos showed a strong feeding preference for humans as did sandflies for armadillos, thus presenting potential utility to further study related to host-vector interactions.
\end{abstract}

\section{Introduction}

Rapid and cost-effective biodiversity surveillance is a critical, burgeoning tool in ecology and conservation. DNA metabarcoding, or high-throughput DNA sequencing of taxonomically informative barcode genes, has become a well-established tool to efficiently detect many species from bulk DNA samples for biodiversity surveillance. Such sequencing is now frequently used to catalog species from the DNA that organisms shed into the environment (eDNA) (Bohmann et al., 2014). For example, aquatic vertebrate or invertebrate communities can be detected with eDNA metabarcoding of water samples (Shaw et al., 2016; Stat et al., 2017; Valentini et al., 2016), animal diets can be detected from eDNA metabarcoding of feces (De Barba et al., 2014; Massey et al., in press; Roffler et al., 2021), and plants and fungi can be detected by eDNA metabarcoding of pollen or ice core samples (Bell et al., 2019; Kraaijeveld et al., 2015; Varotto et al., 2021). Similarly, an emerging technique to monitor the diversity of terrestrial vertebrates uses animalfeeding invertebrates (whether feeding upon blood, flesh, or scat) as direct sources of species' DNA and is called invertebrate-derived DNA (iDNA) (Batovska et al., 2018; Calvignac-Spencer et al., 2013; Drinkwater et al., 2019, 2020; Gogarten et al., 2020; Kocher et al., 2017; P. S. Lee et al., 2016; Schnell et al., 2012; van der Heyde et al., 2020; D. W. Yu et al., 2012). Similar to camera traps, a conventional method for terrestrial 
vertebrate surveillance, metabarcoding blood, carrion, or fecal meals from iDNA samplers can be utilized as a non-invasive and efficient method at profiling a diversity of species in often remote or dense habitats. However, these methods differ in that camera trapping is strongly biased for the detection of medium to large terrestrial mammals, with lower detection probabilities of arboreal species, smaller bodied species, and other non-mammal taxa (Burton et al., 2015; O'Brien \& Kinnaird, 2011). These drawbacks could make iDNA metabarcoding a more attractive method for biodiversity monitoring, particularly if iDNA is better able to detect the birds, amphibians, reptiles, small mammals, and arboreal mammals that camera trapping often fails to detect.

Leeches (Drinkwater et al., 2019; Schnell et al., 2012, 2018; Weiskopf et al., 2018) and carrion flies (CalvignacSpencer et al., 2013; P. S. Lee et al., 2016; Lynggaard et al., 2019; Rodgers et al., 2017) have been the primary sources of validating iDNA metabarcoding for monitoring vertebrate biodiversity. The limited number of studies utilizing metabarcoding with leeches or carrion flies as iDNA samplers have shown that both taxa effectively profile biodiversity in remote or difficult to access environments such as tropical forests (Drinkwater et al., 2020; Gogarten et al., 2020; P. S. Lee et al., 2016; Weiskopf et al., 2018). There is a growing literature of comparing carrion flies as iDNA sources for metabarcoding to camera trapping (Gogarten et al., 2020; P. S. Lee et al., 2016; Rodgers et al., 2017) but even with their utility for profiling animal diversity, neither leeches nor carrions flies are widely collected for other research purposes.

In contrast, some hematophagous insects such as mosquitos and sandflies are important pathogen vectors worldwide and are routinely sampled for disease surveillance. Should these pathogen vectors be effective samplers of vertebrate biodiversity, then using iDNA to profile biodiversity would be an attractive supplemental and/or complementary pursuit to other study objectives using vector species (Batovska et al., 2018; Kocher et al., 2017). We are aware of only one study that has compared mosquitos and sandflies as iDNA samplers and found that both were seemingly effective a describing vertebrate diversity (Kocher et al., 2017). However, these results were not compared to data from a more established iDNA sampler or to more conventional methods of diversity surveillance.

A direct comparison of the emerging iDNA samplers (vectors such as mosquitos and sandflies) to the commonly utilized iDNA taxa groups (such as carrion flies or leeches) and finally to traditional methods of biomonitoring (such as camera traps) is needed to evaluate the diversity revealed by each iDNA source. Such a comparison will set the groundwork for assessing common biases, benefits, and shortfalls associated with each method given the current lack of comparison of established methods to the emerging iDNA samplers of mosquitos and sandflies. Invertebrate taxa differ in their feeding ecologies and other life history traits, which can introduce taxonomic biases into biodiversity assessments when these taxa are used as iDNA sources particularly when utilizing these methods with small sample sizes or spatial scales. Thus, this comparison is particularly needed at a spatial scale where these methods can be evaluated for both alpha and gamma diversity given the same suite of available species.

Here, we aim to assess the effectiveness of iDNA metabarcoding for biodiversity surveillance using landscape scale, bulk sampling of insect groups including carrion flies, sandflies, and mosquitos. We first compare the three iDNA samplers to camera trapping (the established method of vertebrate diversity surveillance) to determine if the two methods reveal similar species richness, composition, and relative abundance across the sampled landscape. Second, we compare the biodiversity found with each of the iDNA samplers to assess the efficiencies and potential biases with using different sources of iDNA as biomonitoring tools. To achieve these comparisons, we examine both gamma and alpha diversity with each iDNA sampler and tease apart the likely causes of dissimilarity.

\section{Materials and Methods}

\section{Study area}

This study region near Sinop, Mato Grosso, Brazil (-1204'73.9"S -55deg43'85.0"W) represents topography and vegetation typical of the ecotone between the Cerrado (dominated by shrubland and grassland) and Amazonia (tropical forests) biomes (Mittermeier et al., 2003). The southern border of the Amazon rain- 
forest was once contiguous dry, tropical forest that has been rapidly deforested by agricultural activity and urbanization. This has resulted in a checkerboard-like pattern of forest and agricultural patches (Fig. 1) with a mix of other matrix types including urban areas and secondary vegetation.

The area is largely flat and dominated by a hot and humid climate with daily temperatures showing greater fluctuation than annual mean low (23degC in June) and high (26degC in September) temperatures. The climate is dominated by dry (May to October) and wet (November to April) seasons with monthly precipitation averages ranging from a low of $2 \mathrm{~mm}$ in July to a high of $310 \mathrm{~mm}$ in February (climate data obtained to Sinop municipality).

\section{Insect sampling}

We collected bulk arthropods (sandflies, mosquitos, and carrion flies) in a coordinated effort at 39 fragmented forest sites of varying size, shape, and degree of fragmentation from April - September in 2015 and 2016 (Fig. 1). At each site, we built a grid of three parallel $200 \mathrm{~m}$ transects with each transect approximately 50 m apart (see Appendix S1: Fig. S1 for trapping grid layout). Along each transect, we placed nine UV LED CDC light traps (BioQuip; Catalog Number: 2770) with approximately $30 \mathrm{~m}$ between traps for 27 total light traps per site. We also set three homemade carrion fly traps at each grid in a triangular arrangement with one trap at the end of the first transect, one at the beginning of the second transect, and the final trap at the end of the third transect (Appendix S1: Fig. S1). We engineered each carrion fly trap from two 2 $\mathrm{L}$ soda bottles and gauze mesh. The trap was designed to bait carrion flies with the smell of rotting meat while also keeping the trapped carrion flies separated from the bait (see Appendix S1: Fig. S2 for carrion fly trap design). We trapped and collected the insects over the course of 4 days and 3 trap nights at each site. We checked traps each day and collected the collection cups containing the live insects (and replaced this with a new collection cup) every 24 hours; we placed the collection cups containing the live insects in a portable refrigerator during transportation to Universidade Federal de Mato Grosso (UFMT, Sinop campus) lab facilities. We immediately transferred insect collections to a $-20 \operatorname{deg} C$ freezer for at least 30 minutes to stun the insects in order to then separate out sandflies and mosquitos from other insect by-catch. We sorted sandflies, mosquitos, and carrion flies into separate pools based on site and date into $2 \mathrm{~mL}$ microtubes. Because of differences in body size and weight, sandflies were pooled at 50 individuals, mosquitos were pooled at fifteen individuals, and carrion flies were pooled at five individuals. Finally, we placed sorted insects into a -80degC freezer until they were shipped using dry ice to our home lab facility at Oregon State University where they were once again frozen at $-80 \mathrm{deg} \mathrm{C}$ until molecular processing.

\section{DNA extraction, amplification, and sequencing}

Prior to DNA extraction, we macerated pooled samples using ceramic beads (sandflies), disposable tissue grinding pestles (mosquitos), and manual maceration using the blunt-end of a disposable tipped applicator (carrion flies). We extracted DNA from sandflies and carrion flies with the Qiagen Blood and Tissue Kit (Qiagen, Hilden, Germany) with slight modifications to the manufacturer's specifications. Briefly, $200 \mathrm{uL}$ of Buffer ATL and $20 \mathrm{uL}$ of Proteinase $\mathrm{K}$ were added to the sample in a $1.7 \mathrm{~mL}$ Eppendorf tube and the sample incubated for 3-5 hours at 56degC. Post-incubation, samples were vortexed for 10 minutes and then purified through washing. The DNA was eluted in a final volume of $100 \mathrm{uL}$. Because we wanted to isolate both DNA and RNA from mosquitos for separate studies of viral surveillance, we extracted nucleic acids following a phenol-chloroform protocol modified from Griffiths et al. (2000) and Simister et al. (2011) with final ethanol precipitation and purification to isolate DNA. To purify for DNA, we resuspended the nucleic acid pellet in $50 \mathrm{uL}$ of RNase-free water and incubated half the sample $(25 \mathrm{uL})$ with RNase A in a final concentration of $100 \mathrm{ug} / \mathrm{mL}$ for 10 minutes at $37 \mathrm{degC}$. The other half of the sample was purified for RNA and then synthesized for cDNA.

We amplified the extracted DNA from each iDNA sampler type in two separate reactions using a slight modification of the pan-vertebrate primer pair 12SV5F/12SV5R (Riaz et al., 2011), which targets approximately 100 base pairs in the $12 \mathrm{~S}$ region of the vertebrate mitochondrial genome. We used the reverse primer 12SV5R (TTAGATACCCCACTATGC) as Riaz et al. (2011) and a modified version (we change the thymine to a 
degenerate base shown underlined) of the forward primer 12SV5F to allow for broader binding of vertebrate targets ( $Y$ AGAACAGGCTCCTCTAG). In brief, PCR reactions were carried out in a volume of $20 \mathrm{uL}$ using $10 \mathrm{uL}$ AmpliTaq Gold 360 Master Mix (final concentration of 1x), $5 \mathrm{uL}$ of forward and reverse primers (final concentration of $0.25 \mathrm{uM}$ ), $3 \mathrm{uL}$ of water, and $2 \mathrm{uL}$ of DNA template. PCR cycling was as follows: initial denaturing at $95 \mathrm{deg} \mathrm{C}$ for 10 minutes followed by 40 cycles of $95 \mathrm{degC}$ for 30 seconds and $58 \mathrm{degC}$ for 30 seconds and $72 \mathrm{degC}$ for 30 seconds, and a final extension at $72 \mathrm{degC}$ for 7 minutes.

PCR amplicons were cleaned using PCRClean DX solid-phase reversible immobilization magnetic beads (Aline Biosciences, Woburn, MA, USA). Each PCR reaction was quantified using Accublue High Sensitivity dsDNA Quantitation kit (Biotium, Fremont, CA, USA) and normalized to $6 \mathrm{ng} / \mathrm{uL}$. Each group of 384 PCR products was then pooled into a single library, for a total of two libraries for carrion flies, four libraries for sandflies, and two libraries for mosquitos. Individual libraries were then tagged with an additional 6 base pair identifying index using the NEBnext Ultra II DNA Library Prep kit (New England Biolabs, Ipswich, MA, USA). Pooled samples were analyzed on a bioanalyzer to confirm fragment size. The libraries were then sequenced using the Illumina HiSeq 3000 (Illumina, San Diego, CA, USA) at the Center for Genome Research and Biocomputing at Oregon State University.

\section{Sequence analysis}

We analyzed raw sequence reads using a bioinformatics pipeline designed to trim and sort the sequence reads according to scat sample identification. An outline of the bioinformatic process is as follows: (1) raw reads were paired using PEAR software (Zhang et al., 2014); (2) followed by demultiplexing using 8 basepair index sequences unique to each sample (mismatches discarded) using a novel grep regular expression; (3) lastly, OTUs from each sample were taxonomically assigned using BLAST against 12S vertebrate sequences available in GenBank.

We carried out a series of filtering and quality control measures on taxonomically assigned sequences. For each of the three iDNA datasets, we removed contaminant reads (primarily human DNA sequences) and removed sample replicates that did not amplify (below a 500 read threshold). We then removed OTU's with either a percent identity score less than $95 \%$ or $1 \%$ of the total number of sequences in that sample. Finally, we eliminated species that were not found in both sample replicates. We then manually reviewed BLAST results for each purported species to ensure that the $12 \mathrm{~S}$ barcode discriminated species from sympatric congeners or confamilials and to confirm that the taxonomic assignments were for species regional to Mato Grosso, Brazil. If species were not regional, we examined the other equal matches to reassign non-regional species. If no suitable species level matches were discovered, then these taxa were assigned at the genus level or removed from the dataset.

\section{Camera traps}

To compare metabarcoding results to methods traditionally used for profiling animal abundance and/or occurrence, we used digital infrared camera traps $\left(\right.$ Bushnell $^{(\mathrm{r})}$ ) to sample vertebrate diversity from May 2015 - March 2016 at 33 of the 39 sites where we collected arthropods as iDNA sources. In total, we deployed 116 camera traps for a total of 5,093 camera days and cameras per site varied from two to seven although at the majority of sites three cameras were utilized. We set up the camera traps $40 \mathrm{~cm}$ above the ground and they were active 24 -hours per day. Camera days per camera ranged from $26-92$ with the average number of camera days equal to 44 days.

\section{Data analysis}

Sample-size-based rarefaction curves with extrapolation (Chao et al., 2014) were created using the iNEXT package in R (Hsieh et al., 2016) to examine species richness across the three iDNA datasets and from the camera trapping data. For the iDNA data, we converted species read abundance to species incidence data (presence/absence) at the sample level. Due to differences in the number of amplified samples across the three iDNA sources, species richness was extrapolated to the iDNA source with the greatest number of samples following Chao et al. (2014). For the camera trapping data, we measured species richness across 
the total number of camera days from all deployed cameras.

We used a relative abundance index (RAI) to compare the biodiversity found using iDNA metabarcoding data and camera trap data. For metabarcoding data, the RAI is equal to the sum of occurrences for species $i$ divided by the total number of pooled samples. The RAI measured from camera trapping data is calculated as the number of species $i$ events divided by the total number of camera trap nights. A single species event from camera trap data was defined by any individual (or group of individuals) from one species captured by a camera over a one-hour time frame. We then used RAI to visualize the gamma diversity according to both iDNA and camera trapping methods by binning species according to important life history traits to compare the effectiveness of the biomonitoring methods in describing the gamma diversity of different taxa groups. To compare RAI across iDNA and camera traps, we only included sites that were sampled by both methods.

After comparing diversity from iDNA and camera traps, we then compared the iDNA sources to each other to assess the similarities, efficiencies, and potential biases of each sampler type. We used a Bray-Curtis dissimilarity matrix of sites $\mathrm{x}$ species and applied a PERMANOVA test using the adonis function to test for significance in the separation of the vertebrate community based on iDNA sampler. We then used the vegan package in $\mathrm{R}$ (Oksanen et al., 2013) and themetaMDS function to visualize dissimilarities in species composition at sites using non-metric multidimensional scaling (NMDS). Each site was designated three rows in our abundance matrix: one for each iDNA type (carrion fly, sandfly, or mosquito). We also utilized theordiellipse function to visualize the spread of the data based on iDNA source with ellipses drawn to two standard deviation (95\% confidence intervals) from the group mean. Lastly, significant species vectors were calculated using the envfit function.

To answer whether an iDNA sampler could replace another iDNA sampler in profiling alpha diversity, we calculated RAI of species at the site level for each of the iDNA datasets and compared species occurrences at sites from the different iDNA samplers. We visualized the results in scatterplots. If two iDNA datasets showed similar species composition at sites, most data points would fall along an isometric line. If two iDNA samplers revealed dissimilar species composition across sites, more data points would fall along the x-axis or y-axis revealing occurrence of a species in one of the iDNA datasets and an absence of that species in the other iDNA dataset. Finally, to further explore the different feeding ecologies associated with the insect taxa used as iDNA samplers, we compared the RAI at sites for the most abundant species in each iDNA dataset using boxplots.

\section{Human DNA}

When analyzing metabarcoding data for profiling vertebrate diversity, we remove all human DNA reads under the assumption that they are contamination. However, we found a disproportionate amount of the DNA sequences from mosquito pools was identified to be human and the total human read abundance in samples was well above the level of human DNA found in the extraction and PCR negative controls. Thus, we reexamined levels of human DNA found in all three iDNA metabarcoding datasets. We first eliminated non-human contaminants and non-amplifying samples (replicates with less than 500 total reads). With the remaining dataset, we culled all non-human read sequences. We closely examined the amount of human DNA in the extraction and PCR negative controls to determine a read threshold for a human positive sample. To be extremely conservative in what we deemed as a sample positive for human, we set the threshold as the highest read count in any negative control replicate for each iDNA dataset. For example, if the highest read count for human DNA in a negative control for the carrion fly metabarcoding data was 25,000 then the threshold to be counted as a sample positive for human DNA was 25,000 reads in a replicate. Additionally, we also eliminated samples where human DNA was not present in both sample replicates. A relative abundance index (RAI) was calculated as the number of samples positive for human DNA at a site divided by the total number of samples at a site.

\section{Results}

Carrion fly $12 \mathrm{~S}$ metabarcoding resulted in 16,130,638 paired sequence reads from 368 pooled samples. The 
average read depth per sample replicate was 21,976 reads. The sandfly $12 \mathrm{~S}$ metabarcoding results showed 25,093,673 paired sequence reads from 943 pooled samples and the average read depth per sample replicate is 13,780 reads. Finally, mosquito $12 \mathrm{~S}$ metabarcoding results resulted in $35,132,064$ total paired sequence reads from 322 pooled samples with an average read depth per sample replicate of 55,152 reads.

After conservative quality measures designed to clean the raw data, the following datasets were used for analysis (and excluded human DNA): carrion fly data had 5,010,458 total paired sequences from 265 pooled samples which represented 37 of the 39 sites; the sandfly data had 5,233,020 total paired sequences from 354 pooled samples which represented 36 sites; finally, the mosquito data had 6,254,399 paired sequences from 78 pooled samples which represented 25 sites (a summary of site-level data for each iDNA method can be found in Appendix S1: Table S1). Pooled samples of carrion flies showed the highest amplification success followed by pooled sandfly samples and finally pooled mosquito samples (Table 1), although these results exclude human DNA which made up the majority of sequence reads in the mosquito dataset.

\section{Comparing biodiversity found with insect samplers and camera traps}

In total, iDNA and camera trapping methods revealed 90 vertebrate species across the sampled landscape (Table 2). iDNA alone described 82 species with carrion fly data describing the most diversity of any sampler with 62 species while sandfly data revealed 49 species and mosquito data revealed 16 species. Camera trap data revealed 28 vertebrate species and added 8 species to the diversity already described with iDNA.

Species accumulation curves show a statistically significant difference in species richness gleaned from each iDNA source and camera trapping (Fig. 2). Carrion fly data and mosquito data are extrapolated to match the number of sandfly samples, and consequently the extrapolated curves provide less certainty than the interpolated curves. However, the confidence intervals show significant separation in the total species richness from each iDNA source across both the interpolated and extrapolated curves. Using carrion flies as a measure of biodiversity reveals a significantly greater number of vertebrate species across the landscape than both sandflies and mosquitos. Additionally, the total number of carrion flies analyzed $(\mathrm{n}=1,759)$ was lower than both the total number of sandflies $(\mathrm{n}=48,686)$ and mosquitos $(\mathrm{n}=4,776)$ analyzed. The species accumulation curve for camera trap data shows that species richness plateaus after approximately 1,500 camera days meaning that few to no new species are revealed by camera traps even with continued camera days (Fig. 2). The species richness from camera traps was most similar to the species richness from the extrapolated curve for the pooled mosquito samples, which was the least efficient iDNA sampler in describing vertebrate diversity (Fig. 2; Table 1).

Comparisons of species' RAI from iDNA samplers and camera traps show differences in diversity profiles across taxa groups (Fig. 3). As expected, camera traps showed a high diversity of carnivore and ungulate species and a low diversity of arboreal species or birds. Camera traps also did not capture the occurrence or diversity of domestic species and rodent species particularly when compared to the diversity of these groups found using iDNA methods. Carrion fly data consistently showed a high diversity of species in each taxa group. Sandfly data also showed high diversity of species in most taxa groups with an especially high diversity and relative abundance of armadillo species (Fig. 3; Table 2). Lastly, mosquito data showed the lowest species diversity for many of the taxa groups and only showed a high diversity of domestic species when comparing mosquito data to the other datasets (although carrion fly data still displayed greater diversity and relative abundance from domestic species). When species incidence data was parsed at the site level, camera traps showed the highest median species richness at sites (Fig. 4) and a consistent diversity profile across sites (Appendix S1: Fig. S3) indicating that camera trapping consistently samples the same species across sites.

\section{Comparing the different iDNA samplers for biomonitoring}

A PERMANOVA test and further visualization of the dissimilarity matrix using NMDS $(\mathrm{k}=2$; stress $=$ 0.17 ) show that the gamma diversity did not differ significantly based on iDNA source ( $\mathrm{p}$-value $=0.23$ ) (Fig. 5). Although there was no statistically significant separation in the gamma diversity found using the different iDNA methods, a site-by-site comparison of the alpha diversity found with each iDNA method does 
not support substantial overlap of the vertebrate communities revealed by each iDNA source at the site-level (Fig. 5). Although carrion flies revealed the greatest gamma diversity, when comparing species presence at a site across iDNA datasets, we found that there were many species absences in the carrion fly data at sites where sandflies or mosquito samplers recorded a species presence (Appendix S1: Fig. S4). Carrion fly data missed 181 of the 213 species occurrences from the sandfly data and 42 of the 63 total species occurrences from the mosquito data when examined at the site-level (Appendix S1: Fig. S4). Arrowed vectors show that the significant species in the ordination ( $\mathrm{p}$-value $<0.001$ ) were also the most abundant in each iDNA dataset, and thus these species likely drive the separation in the vertebrate communities at sites as described by the different iDNA samplers (Fig. 5). These significant, non-human vertebrate species include cattle (highest RAI in carrion fly data), dog (highest RAI in mosquito data), nine-banded armadillo (highest RAI in sandfly data), capuchin, lesser anteater, and turkey (second highest RAI in mosquito data). This sampler bias was confirmed by our analysis of the most abundant species in each of the iDNA datasets which revealed the differing feeding associations of each insect group (Fig. 6) and we found that these abundant species were responsible for many of the site-level mismatches between the iDNA samplers (Appendix S1: Fig. S4). Additionally, we determined that humans were the primary feeding target of mosquitos (Fig. 6) and human DNA accounted for more than $80 \%$ of the total sequence reads in the mosquito iDNA dataset.

\section{Discussion}

This study provides evidence that carrion flies are the superior method for landscape scale biodiversity surveillance compared to both camera traps and the other iDNA sampling methods (carrion fly iDNA identified 62 vertebrate species, sandfly iDNA detected 49 species, mosquito iDNA detected 16 species, and camera traps identified 28 species). Previous work has found that iDNA from carrion flies produced complementary results when compared to more traditional methods of biomonitoring such as camera traps (Gogarten et al., 2020; Rodgers et al., 2017). We found a similar pattern to Rodgers et al. (2017) in their comparison of carrion flies to existing camera trap data in that even with a small sample size and effort, carrion flies used as iDNA samplers were able to profile most of the diversity that had been found with longterm camera trapping while also revealing new species diversity. However, with our larger sampling effort, we were able to show that carrion flies were much more effective at describing diversity at the landscape scale and species richness likely would have increased for each iDNA sampler if we had increased our sampling effort, particularly at the site level, whereas species richness had fully saturated by 1,500 camera-days (Fig. 2 and Fig. 4).

While camera traps revealed lower gamma diversity than carrion flies and sandflies, they had the greatest median species richness at sites (alpha diversity) compared to each of the iDNA samplers. This points to camera traps reliably revealing the same suite of species from across sites resulting in higher alpha diversity but lower overall gamma diversity when compared to iDNA samplers. The missing species richness exhibited by camera traps at the landscape scale of our study is likely due to camera traps preferentially targeting larger-bodied mammals (Burton et al., 2015; O'Brien \& Kinnaird, 2011). We found support for this as camera traps revealed the greatest diversity of carnivore species and ungulate species while missing most of the iDNA-described diversity of the arboreal species and the smaller-bodied mammals.

The improved biodiversity detection with carrion flies over other iDNA sources and camera traps comes with additional logistical advantages. Carrion flies can be trapped with inexpensive, homemade traps compared to the more expensive camera traps or the UV LED CDC light traps used to capture sandflies and mosquitoes. These homemade traps also did not require batteries compared to the UV LED CDC light traps which require $6 \mathrm{~V}$ batteries that need to be changed approximately every 24 hours. Our trap design (Appendix S1: Fig. S2) was effective at attracting and trapping carrion flies while at the same time preventing individuals from accessing the bait. Using a blocking primer for the species used as bait is also an option when utilizing carrion flies as an iDNA source. Carrion flies are thus much more amenable to sampling in remote landscapes where transporting and shipping pre-made traps or bulky equipment can be difficult. We also identified the most species with carrion flies compared to the other iDNA samplers despite a much lower sampling effort of 3 traps per site compared to 27 traps per site for mosquitos and sandflies, which resulted in much fewer individual 
carrion flies $(\mathrm{n}=1,759)$ compared to the total number of sandflies $(\mathrm{n}=56,774)$ and mosquitos $(\mathrm{n}=4,776)$. Carrion fly samples had the greatest amplification efficiency of non-human vertebrates (Table 1) despite only 5 individual flies coextracted compared with 15 mosquitos and 50 sandflies, although amplification efficiency reflected all captured sandflies (not only blood-fed females) and did not include human DNA which was highest in mosquito samples.

Although carrion flies were the most effective iDNA sampler, we wanted to examine whether the gamma and alpha diversity described varied substantially among iDNA sources to assess the effectiveness of sandflies and mosquitos as biodiversity samplers. We found that the overlap in vertebrate communities from each iDNA source was generally supported at the landscape scale, however, the different iDNA sources detected different communities of species at each site, and the same sites were typically distant in ordination space. Although carrion flies were the most effective sampler for detecting gamma diversity, we found carrion flies were less redundant with mosquitos and sandflies for detecting local biodiversity and carrion flies missed $85 \%$ and $67 \%$ of the species occurrences from across all sites in the sandfly data and in the mosquito data respectively, although most of these misses corresponded to the dominant species in either the sandfly or mosquito data as shown in Appendix S1: Fig. S4. This points to needing a substantially larger sampling effort at the site level for iDNA methods, particularly carrion flies, to saturate species detections.

Aside from the effect of sampling effort on diverging alpha diversity profiles, some of the mismatch is explained by the differing feeding ecologies of each insect group. We detected substantial taxonomic biases in vertebrate detections among iDNA sources that were likely driven by differences in the host preferences of the common mosquito and sandfly species. Aside from domestic dog, we found evidence of contrasting feeding preferences for vector taxa that were consistent with to their known relationships with host species. Sandflies overwhelmingly targeted armadillos (Dasypus novemcinctus and Dasypus kappleri) which aligns with our understanding of both the relationship between armadillos and sandflies (Akhoundi et al., 2016; Alexander, 2000; Lainson et al., 1979) as well as armadillos as an important host species for the life cycle of Leishmania parasites (Lainson et al., 1979; Lainson \& Shaw, 1989), which are transmitted by female sandfly vectors. Kocher et al. (2017) reported the first direct evidence of sandfly-armadillo interaction using metabarcoding of sandflies, and our findings overwhelmingly support this finding. Mosquitos also exhibited strong host preferences for humans, which even after rigorous quality control to eliminate contamination was the dominant iDNA data source representing $80 \%$ of the total reads. While many species of mosquito are opportunistic feeders, there are a number of species that target humans including Aedes aegypti which is the main vector for dengue, yellow fever, and chikungunya and is found throughout tropical and subtropical regions worldwide (McBride, 2016; Zwiebel \& Takken, 2004). Interestingly, our metabarcoding data is corroborated by previous studies spanning the Amazon region that show upwards of $80 \%$ of $A$. aegypti bloodmeals contain human DNA (McBride, 2016). Compared to the targeted feeding behavior of sandflies and mosquitos (as well as leeches shown by Drinkwater et al. 2019), the feeding ecology of carrion flies may offer the least biased view of the diversity and relative biomass of species across a landscape because their feeding behavior is likely driven by both the presence of carrion and scat (Calvignac-Spencer et al., 2013; Rodgers et al., 2017). Given these findings, the feeding ecologies of each insect group should be an important consideration because choice of iDNA sampler can lead to mismatches in the vertebrate diversity profiles, especially with smaller sampling sizes.

By exploring the biases associated with each sampling type, we found evidence that carrion flies are significantly more effective in describing landscape level biodiversity when compared to diversity found with camera traps and other iDNA samplers, even with the least sampling effort and smallest sample size. We found that camera traps predictably were biased towards larger-bodied mammals and away from arboreal and smaller-bodied species, thus missing much of the biodiversity described with iDNA samplers, but also had the highest species richness at the site-level. Sandflies and mosquitos show feeding behavior targeted at armadillos and humans, respectively. Although sandflies and mosquitos were inferior samplers for large-scale biodiversity surveillance compared to carrion flies, they add value to existing host-vector-pathogen surveillance efforts. This is particularly relevant in forest frontiers where vectors maintain enzootic sylvatic cycles which can cause disease spillover into humans and/or domestic animals if they are bitten by these infected 
vectors (Figueiredo, 2007; Vasconcelos et al., 2001). Additionally, our findings support the known ecological relationships between these vector taxa and their hosts thus further validating metabarcoding results within the larger realm of ecological research. With the advances in high-throughput sequencing and the steadily decreasing expense of metabarcoding, iDNA should be used as an effective and efficient method for biomonitoring.

\section{Acknowledgements}

Support for this research was funded by The British Council Department of Business, Innovation and Skills through their Global Innovation Initiative and through Oregon State University. We thank everyone who assisted with establishing sites, with data collection, and with sample processing including C.J.S.P. Vieira, J.R. Kubiszeski, C. Benishek, S. Schmidt, and A. Pepper. We would also like to acknowledge the landowners across Sinop, Mato Grosso who allowed us access to their properties for insect collection and camera trapping. The Center for Genome Research and Biocomputing (CGRB) at Oregon State University provided access to the Illumina HiSeq 3000 for all metabarcoding. We also thank the Levi lab members for reviewing early versions of this manuscript.

\section{References}

Akhoundi, M., Kuhls, K., Cannet, A., Votypka, J., Marty, P., Delaunay, P., \& Sereno, D. (2016). A historical overview of the classification, evolution, and dispersion of Leishmania parasites and sandflies.PLoS Neglected Tropical Diseases , 10 (3), e0004349.

Alexander, B. (2000). Sampling methods for phlebotomine sandflies.Medical and Veterinary Entomology, $14(2), 109-122$.

Batovska, J., Lynch, S. E., Cogan, N. O. I., Brown, K., Darbro, J. M., Kho, E. A., \& Blacket, M. J. (2018). Effective mosquito and arbovirus surveillance using metabarcoding. Molecular Ecology Resources ,18 (1), $32-40$.

Bell, K. L., Burgess, K. S., Botsch, J. C., Dobbs, E. K., Read, T. D., \& Brosi, B. J. (2019). Quantitative and qualitative assessment of pollen DNA metabarcoding using constructed species mixtures. Molecular Ecology , 28 (2), 431-455.

Bohmann, K., Evans, A., Gilbert, M. T. P., Carvalho, G. R., Creer, S., Knapp, M., Douglas, W. Y., \& De Bruyn, M. (2014). Environmental DNA for wildlife biology and biodiversity monitoring. Trends in Ecology and Evolution, 29 (6), 358-367.

Burton, A. C., Neilson, E., Moreira, D., Ladle, A., Steenweg, R., Fisher, J. T., Bayne, E., \& Boutin, S. (2015). Wildlife camera trapping: A review and recommendations for linking surveys to ecological processes. Journal of Applied Ecology , 52 (3), 675-685.

Calvignac-Spencer, S., Merkel, K., Kutzner, N., Kuhl, H., Boesch, C., Kappeler, P. M., Metzger, S., Schubert, G., \& Leendertz, F. H. (2013). Carrion fly-derived DNA as a tool for comprehensive and cost-effective assessment of mammalian biodiversity. Molecular Ecology ,22 (4), 915-924.

Chao, A., Gotelli, N. J., Hsieh, T. C., Sander, E. L., Ma, K. H., Colwell, R. K., \& Ellison, A. M. (2014). Rarefaction and extrapolation with Hill numbers: A framework for sampling and estimation in species diversity studies. Ecological Monographs , 84 (1), 45-67.

De Barba, M., Miquel, C., Boyer, F., Mercier, C., Rioux, D., Coissac, E., \& Taberlet, P. (2014). DNA metabarcoding multiplexing and validation of data accuracy for diet assessment: Application to omnivorous diet. Molecular Ecology Resources , 14 (2), 306-323.

Drinkwater, R., Jucker, T., Potter, J. H. T., Swinfield, T., Coomes, D. A., Slade, E. M., Gilbert, M. T. P., Lewis, O. T., Bernard, H., Struebig, M. J., Clare, E. L., \& Rossiter, S. J. (2020). Leech blood-meal iDNA reveals differences in Bornean mammal diversity across habitats. Molecular Ecology . 
Drinkwater, R., Schnell, I. B., Bohmann, K., Bernard, H., Veron, G., Clare, E. L., Gilbert, M. T. P., \& Rossiter, S. J. (2019). Using metabarcoding to compare the suitability of two blood-feeding leech species for sampling mammalian diversity in North Borneo.Molecular Ecology Resources , 19 (1), 105-117.

Figueiredo, L. T. M. (2007). Emergent arboviruses in Brazil.Revista Da Sociedade Brasileira de Medicina Tropical ,40 (2), 224-229.

Gogarten, J. F., Hoffmann, C., Arandjelovic, M., Sachse, A., Merkel, K., Dieguez, P., Agbor, A., Angedakin, S., Brazzola, G., Jones, S., Langergraber, K. E., Lee, K., Marrocoli, S., Murai, M., Sommer, V., Kuhl, H., Leendertz, F. H., \& Calvignac-Spencer, S. (2020). Fly-derived DNA and camera traps are complementary tools for assessing mammalian biodiversity. Environmental DNA, 2 (1), 63-76.

Griffiths, R. I., Whiteley, A. S., O'Donnell, A. G., \& Bailey, M. J. (2000). Rapid method for coextraction of DNA and RNA from natural environments for analysis of ribosomal DNA-and rRNA-based microbial community composition. Applied and Environmental Microbiology ,66 (12), 5488-5491.

Hsieh, T. C., Ma, K. H., \& Chao, A. (2016). INEXT: an R package for rarefaction and extrapolation of species diversity (Hill numbers).Methods in Ecology and Evolution , 7 (12), 1451-1456.

Kocher, A., de Thoisy, B., Catzeflis, F., Valiere, S., Banuls, A.-L., \& Murienne, J. (2017). iDNA screening: Disease vectors as vertebrate samplers. Molecular Ecology , 26 (22), 6478-6486.

Kraaijeveld, K., De Weger, L. A., Ventayol Garcia, M., Buermans, H., Frank, J., Hiemstra, P. S., \& Den Dunnen, J. T. (2015). Efficient and sensitive identification and quantification of airborne pollen using nextgeneration DNA sequencing. Molecular Ecology Resources ,15 (1), 8-16.

Lainson, R., \& Shaw, J. J. (1989). Leishmania (Viannia) naiffi sp. N., a parasite of the armadillo, Dasypus novemcinctus (L.) in Amazonia Brazil. Annales de Parasitologie Humaine et Comparee ,64 (1), 3-9.

Lainson, R., Shaw, J. J., Ward, R. D., Ready, P. D., \& Naiff, R. D. (1979). Leishmaniasis in Brazil XIII: isolation of Leishmania from armadillos (Dasypus novemcinctua), and observations on the epidemiology of cutaneous leishmaniasis in north Para State. Transactions of the Royal Society of Tropical Medicine and Hygiene , 73 (2), 239-242.

Lee, P. S., Gan, H. M., Clements, G. R., \& Wilson, J. J. (2016). Field calibration of blowfly-derived DNA against traditional methods for assessing mammal diversity in tropical forests. Genome ,59 (11), 1008-1022.

Lynggaard, C., Nielsen, M., Santos-Bay, L., Gastauer, M., Oliveira, G., \& Bohmann, K. (2019). Vertebrate diversity revealed by metabarcoding of bulk arthropod samples from tropical forests. Environmental DNA ,1 (4), 329-341.

Massey, A. L., Roffler, G. H., Vermuel, T., Allen, J., \& Levi, T. (in press). Comparison of mechanical sorting and DNA metabarcoding for diet analysis with fresh and degraded wolf scats. Ecosphere .

McBride, C. S. (2016). Genes and odors underlying the recent evolution of mosquito preference for humans. Current Biology, 26 (1), R41-R46.

Mittermeier, R. A., Mittermeier, C. G., Brooks, T. M., Pilgrim, J. D., Konstant, W. R., Da Fonseca, G. A., \& Kormos, C. (2003). Wilderness and biodiversity conservation. Proceedings of the National Academy of Sciences , 100 (18), 10309-10313.

O'Brien, T. G., \& Kinnaird, M. F. (2011). Estimation of species richness of large vertebrates using camera traps: An example from an Indonesian rainforest. In Camera Traps in Animal Ecology (pp. 233-252). Springer.

Oksanen, J., Blanchet, F. G., Kindt, R., Legendre, P., Minchin, P. R., O'hara, R. B., Simpson, G. L., Solymos, P., Stevens, M. H. H., Wagner, H., \& Oksanen, M. J. (2013). Package "vegan." Community Ecology Package, Version, 2 (9), 1-295. 
Riaz, T., Shehzad, W., Viari, A., Pompanon, F., Taberlet, P., \& Coissac, E. (2011). ecoPrimers: Inference of new DNA barcode markers from whole genome sequence analysis. Nucleic Acids Research ,39 (21), e145-e145.

Rodgers, T. W., Xu, C. C., Giacalone, J., Kapheim, K. M., Saltonstall, K., Vargas, M., Yu, D. W., Somervuo, P., McMillan, W. O., \& Jansen, P. A. (2017). Carrion fly-derived DNA metabarcoding is an effective tool for mammal surveys: Evidence from a known tropical mammal community.Molecular Ecology Resources , 17 (6), e133-e145.

Roffler, G. H., Allen, J., Massey, A. L., \& Levi, T. (2021). Metabarcoding of fecal DNA shows dietary diversification in wolves substitutes for ungulates in an island archipelago. Ecosphere ,12 (1), e03297.

Schnell, I. B., Bohmann, K., Schultze, S. E., Richter, S. R., Murray, D. C., Sinding, M. H. S., Bass, D., Cadle, J. E., Campbell, M. J., Dolch, R., \& Edwards, D. P. (2018). Debugging diversity-A pan-continental exploration of the potential of terrestrial blood-feeding leeches as a vertebrate monitoring tool. Molecular Ecology Resources ,18 (6), 1282-1298.

Schnell, I. B., Thomsen, P. F., Wilkinson, N., Rasmussen, M., Jensen, L. R., Willerslev, E., Bertelsen, M. F., \& Gilbert, M. T. (2012). Screening mammal biodiversity using DNA from leeches. Current Biology, 22 (8), R262-R263.

Shaw, J. L., Clarke, L. J., Wedderburn, S. D., Barnes, T. C., Weyrich, L. S., \& Cooper, A. (2016). Comparison of environmental DNA metabarcoding and conventional fish survey methods in a river system.Biological Conservation , 197, 131-138.

Simister, R. L., Schmitt, S., \& Taylor, M. W. (2011). Evaluating methods for the preservation and extraction of DNA and RNA for analysis of microbial communities in marine sponges. Journal of Experimental Marine Biology and Ecology, 397 (1), 38-43.

Stat, M., Huggett, M. J., Bernasconi, R., DiBattista, J. D., Berry, T. E., Newman, S. J., Harvey, E. S., \& Bunce, M. (2017). Ecosystem biomonitoring with eDNA: metabarcoding across the tree of life in a tropical marine environment. Scientific Reports, 7 (1), 1-11.

Valentini, A., Taberlet, P., Miaud, C., Civade, R., Herder, J., Thomsen, P., Bellemain, E., Coissac, E., Boyer, F., \& Gaboriaud, C. (2016). Next-generation monitoring of aquatic biodiversity using environmental DNA metabarcoding. Molecular Ecology , 25 (4), 929-942.

van der Heyde, M., Bunce, M., Wardell-Johnson, G., Fernandes, K., White, N. E., \& Nevill, P. (2020). Testing multiple substrates for terrestrial biodiversity monitoring using environmental DNA metabarcoding.Molecular Ecology Resources , 20 (3), 732-745.

Varotto, C., Pindo, M., Bertoni, E., Casarotto, C., Camin, F., Girardi, M., Maggi, V., \& Cristofori, A. (2021). A pilot study of eDNA metabarcoding to estimate plant biodiversity by an alpine glacier core (Adamello glacier, North Italy). Scientific Reports ,11 (1), 1-3.

Vasconcelos, P. F., Travassos da Rosa, A., Rodrigues, S. G., Travassos da Rosa, E. S., Degallier, N., \& Travassos da Rosa, J. F. (2001). Inadequate management of natural ecosystem in the Brazilian Amazon region results in the emergence and reemergence of arboviruses. Cadernos de Saude Publica, 17, S155-S164.

Weiskopf, S. R., McCarthy, K. P., Tessler, M., Rahman, H. A., McCarthy, J. L., Hersch, R., Faisal, M. M., \& Siddall, M. E. (2018). Using terrestrial haematophagous leeches to enhance tropical biodiversity monitoring programmes in Bangladesh. Journal of Applied Ecology ,55 (4), 2071-2081.

Yu, D. W., Ji, Y., Emerson, B. C., Wang, X., Ye, C., Yang, C., \& Ding, Z. (2012). Biodiversity soup: Metabarcoding of arthropods for rapid biodiversity assessment and biomonitoring. Methods in Ecology and Evolution , 3 (4), 613-623. 
Zhang, J., Kobert, K., Flouri, T., \& Stamatakis, A. (2014). PEAR: a fast and accurate Illumina Paired-End reAd mergeR.Bioinformatics , 30 (5), 614-620.

Zwiebel, L. J., \& Takken, W. (2004). Olfactory regulation of mosquito-host interactions. Insect Biochemistry and Molecular Biology , 34 (7), 645-652.

\section{Data Accessibility}

All metabarcoding data collated for analyses will be available as spreadsheets in the Supplemental Information.

\section{Author Contributions}

ALM led project design, fieldwork, laboratory work, analysis, and writing. RB was involved with project conceptualization and fieldwork and laboratory work design. DJFS was instrumental in assisting with fieldwork and data collection. JA helped with early laboratory protocols and library preparation for metabarcoding. PRL and CSSB carried out camera trapping and image processing and provided raw camera trap data. MSF and GRC helped with project conceptualization and design. CP and TL were involved with project conceptualization and project design and provided substantial feedback on the manuscript.

Table 1: Efficiency of each insect taxa as an iDNA sampler used to profile vertebrate diversity from the blood (sandflies and mosquitos) or carrion meals (carrion flies). Human DNA is excluded.

\begin{tabular}{llll}
\hline & carrion flies & sandflies & mosquitos \\
\hline $\begin{array}{l}\text { Total number of } \\
\text { individuals captured (and } \\
\text { processed) }\end{array}$ & $1,759(1,759)$ & $56,774(48,686)$ & $4,776(4,776)$ \\
$\begin{array}{l}\text { Total number of pooled } \\
\text { samples processed for } \\
\text { metabarcoding }\end{array}$ & 370 & 976 & 321 \\
$\begin{array}{l}\text { Total number of } \\
\text { amplified samples used } \\
\text { for analysis post } \\
\text { quality control } \\
\text { measures }\end{array}$ & 265 & 354 & 78 \\
$\begin{array}{l}\text { Percentage of usable } \\
\text { samples }\end{array}$ & $71.6 \%$ & $36.3 \%$ & \\
\hline
\end{tabular}

Table 2: Vertebrate diversity found using iDNA samplers and camera trapping methods. Values show a species' RAI in a given dataset. An "X" indicates that a species was present at a RAI value less than $1 \%$ (a rare species).

\begin{tabular}{lllll}
\hline & & iDNA: carrion fly & iDNA: sandfly & iDNA: \\
\hline Mammals & Mammals & Mammals & Mammals & Mamma \\
Artiodactyla & Artiodactyla & Artiodactyla & Artiodactyla & Artiodac \\
Bos taurus & cattle & $\mathbf{0 . 3 6}$ & $\mathbf{0 . 0 2}$ & $\mathbf{0 . 0 3}$ \\
Mazama americana & red brocket & $\mathbf{0 . 0 1}$ & $\mathbf{0 . 0 1}$ & \\
Mazama gouazoubira & brown brocket & & & $\mathbf{X}$ \\
Mazama nemorivaga & Amazonian brown brocket & $\mathbf{0 . 0 1}$ & $\mathbf{0 . 0 1}$ & \\
Mazama spp. & deer & & $\mathbf{0 . 0 3}$ & $\mathbf{0 . 0 1}$ \\
Ovis aries & sheep & $\mathbf{0 . 0 4}$ & $\mathbf{X}$ &
\end{tabular}




\begin{tabular}{|c|c|c|c|c|}
\hline & & iDNA: carrion fly & iDNA: sandfly & iDNA: $n$ \\
\hline Carnivora & Carnivora & Carnivora & Carnivora & Carnivo \\
\hline Canis lupus familiaris & domestic dog & 0.23 & 0.16 & 0.15 \\
\hline Cerdocyon thous & crab-eating fox & & & \\
\hline Eira barbara & tayra & 0.01 & & \\
\hline Felis catus & domestic cat & 0.01 & 0.01 & 0.01 \\
\hline Leopardus pardalis & ocelot & & & \\
\hline Nasua nasua & coati & 0.03 & $\mathrm{X}$ & $\mathrm{X}$ \\
\hline Panthera onca & jaguar & & & \\
\hline Puma concolor & cougar & & 0.01 & \\
\hline Puma spp. & cougar & & 0.02 & \\
\hline Puma yagouaroundi & jaguarundi & & & \\
\hline Speothos venaticus & bush dog & $\mathbf{X}$ & $\mathbf{X}$ & \\
\hline Chiroptera & Chiroptera & Chiroptera & Chiroptera & Chiropt \\
\hline Micronycteris hirsuta & hairy big-eared bat & & $\mathbf{X}$ & \\
\hline Phyllostomus hastatus & greater spear-nosed bat & $\mathbf{X}$ & $\mathbf{X}$ & \\
\hline Rhogeessa spp. & bat & & & $\mathbf{X}$ \\
\hline Dasypodidae & Dasypodidae & Dasypodidae & Dasypodidae & Dasypo \\
\hline Cabassous unicinctus & southern naked-tailed armadillo & 0.01 & & \\
\hline Dasypus kappleri & greater long-nosed armadillo & & 0.03 & \\
\hline Dasypus novemcinctus & nine-banded armadillo & 0.01 & 0.17 & 0.01 \\
\hline Dasypus septemcinctus & seven-banded armadillo & & 0.01 & \\
\hline Euphractus sexcinctus & six-banded armadillo & 0.01 & 0.01 & \\
\hline Myrmecophaga tridactyla & giant anteater & & $\mathbf{X}$ & $\mathrm{X}$ \\
\hline Priodontes maximus & giant armadillo & & & \\
\hline Didelphimorphia & Didelphimorphia & Didelphimorphia & Didelphimorphia & Didelph \\
\hline Caluromys lanatus & brown-eared woolly opossum & 0.04 & $\mathrm{X}$ & \\
\hline Caluromys philander & bare-tailed woolly opossum & $\mathbf{X}$ & & \\
\hline Didelphis marsupialis & common opossum & 0.01 & 0.01 & \\
\hline Gracilinanus spp. & opossum & 0.01 & & \\
\hline Marmosa murina & common mouse opossum & 0.01 & & \\
\hline Metachirus nudicaudatus & brown four-eyed opossum & 0.01 & $\mathbf{X}$ & \\
\hline Micoureus demerarae & woolly mouse opossum & & $\mathbf{X}$ & \\
\hline Philander opossum & gray four-eyed opossum & & $\mathrm{X}$ & \\
\hline Lagomorpha & Lagomorpha & Lagomorpha & Lagomorpha & Lagomo \\
\hline Sylvilagus spp. & rabbit & & 0.01 & \\
\hline Perissodactyla & Perissodactyla & Perissodactyla & Perissodactyla & Perissoc \\
\hline Tapirus terrestris & Brazilian tapir & 0.09 & 0.03 & 0.01 \\
\hline Pilosa & Pilosa & Pilosa & Pilosa & Pilosa \\
\hline Tamandua tetradactyla & southern anteater & $\mathrm{X}$ & 0.01 & \\
\hline Primates & Primates & Primates & Primates & Primate \\
\hline Alouatta spp. & howler monkey & 0.02 & & \\
\hline Ateles spp. & spider monkey & 0.02 & $\mathrm{X}$ & \\
\hline Cebus spp. & capuchin & 0.11 & 0.01 & 0.01 \\
\hline Chiropotes albinasus & white-nosed saki & & $\mathrm{X}$ & \\
\hline Plecturocebus cupreus & coppery titi & $\mathbf{X}$ & $\mathbf{X}$ & \\
\hline Plecturocebus vieirai & Vieira's titi & & & \\
\hline Rodentia & Rodentia & Rodentia & Rodentia & Rodent \\
\hline Coendou melanurus & black-tailed hairy dwarf porcupine & $\mathbf{X}$ & $\mathbf{X}$ & \\
\hline Coendou prehensilis & Brazilian porcupine & 0.01 & $\mathbf{X}$ & \\
\hline Cuniculus paca & lowland paca & $\mathrm{X}$ & $\mathbf{X}$ & \\
\hline
\end{tabular}




\begin{tabular}{|c|c|c|c|c|}
\hline & & iDNA: carrion fly & iDNA: sandfly & iDNA: $n$ \\
\hline Dactylomys dactylinus & Amazon bamboo rat & $\mathrm{X}$ & & \\
\hline Dasyprocta azarae & Azara's agouti & 0.01 & & 0.01 \\
\hline Hydrochoerus hydrochaeris & capybara & 0.03 & $\mathbf{X}$ & \\
\hline Makalata didelphoides & Brazilian spiny tree-rat & $\mathrm{X}$ & & \\
\hline Mus musculus & house mouse & 0.01 & 0.01 & \\
\hline Oecomys concolor & unicolored rice rat & & $\mathbf{X}$ & \\
\hline Potos flavus & kinkajou & 0.01 & & \\
\hline Proechimys spp. & spiny rat & & $\mathbf{X}$ & $\mathbf{X}$ \\
\hline Pseudoryzomys simplex & Brazilian false rice rat & $\mathbf{X}$ & & \\
\hline Rattus spp. & rat & 0.01 & $\mathbf{X}$ & \\
\hline Sciurus aestuans & Guianan squirrel & & & \\
\hline Reptiles & Reptiles & Reptiles & Reptiles & Reptiles \\
\hline Paleosuchus trigonatus & smooth-fronted caiman & 0.01 & & \\
\hline Amphibians & Amphibians & Amphibians & Amphibians & Amphib \\
\hline Leptodactylus pentadactylus & smoky jungle frog & & $\mathrm{X}$ & \\
\hline Fish & Fish & Fish & Fish & Fish \\
\hline Hemibrycon jabonero & & $\mathrm{X}$ & & \\
\hline Leporinus copelandii & & $\mathbf{X}$ & & \\
\hline Birds & Birds & Birds & Birds & Birds \\
\hline Amazona ochrocephala & yellow-crowned parrot & $\mathbf{X}$ & & \\
\hline bird spp. & & $\mathbf{X}$ & & \\
\hline Buteo spp. & hawk & 0.01 & & \\
\hline Cairina moschata & Muscovy duck & & $\mathbf{X}$ & \\
\hline Cathartes aura & turkey vulture & $\mathbf{X}$ & $\mathbf{X}$ & \\
\hline Coccyzus erythropthalmus & black-billed cuckoo & 0.02 & $\mathbf{X}$ & \\
\hline Coragyps atratus & black vulture & 0.01 & $\mathbf{X}$ & \\
\hline Crotophaga ani & smooth-billed ani & $\mathrm{X}$ & & \\
\hline Crypturellus tataupa & Tataupa tinamou & 0.01 & & \\
\hline Crypturellus undulatus & undulated tinamou & 0.02 & $\mathbf{X}$ & \\
\hline Fringilla spp. & finch & $\mathbf{X}$ & & \\
\hline Gallus gallus & red junglefowl & 0.01 & $\mathbf{X}$ & \\
\hline Lepidocolaptes angustirostris & narrow-billed woodcreeper & 0.01 & & \\
\hline Lepidothrix coronata & blue-crowned manakin & & & $\mathbf{X}$ \\
\hline Meleagris gallopavo & turkey & 0.02 & $\mathbf{X}$ & 0.07 \\
\hline Nyctiphrynus spp. & poorwill & $\mathbf{X}$ & & \\
\hline Oreopholus ruficollis & tawny-throated dotterel & & & $\mathbf{X}$ \\
\hline Piprites chloris & wing-barred piprites & $\mathbf{X}$ & & \\
\hline Psophia viridis & dark-winged trumpeter & & $\mathbf{X}$ & \\
\hline Ramphastos tucanus & white-throated tucan & & $\mathbf{X}$ & \\
\hline Rhea americana & greater rhea & 0.02 & & \\
\hline Sakesphorus luctuosus & glossy antshrike & 0.01 & $\mathbf{X}$ & \\
\hline tanager spp. & tanager & 0.02 & & \\
\hline Tiaris olivaceus & yellow-faced grassquit & 0.01 & & \\
\hline tinamou spp. & tinamou & 0.01 & & \\
\hline Trogon viridis & green-backed trogon & $\mathrm{X}$ & & \\
\hline
\end{tabular}

Figure 1: Map of the study region centered at Sinop, Mato Grosso, Brazil (shown in purple). The area shaded in green is designated as forest with our study sites labeled as pink circles with an example of the typical landscape at a forested site shown in the photo. We sampled a total of 39 sites using UV LED 
CDC light traps (for sandflies and mosquitos) and 3 carrion fly traps and at 33 of these sites we also used camera traps. The inset map of Brazil shows the Amazonia biome highlighted in green with our study area highlighted in pink.

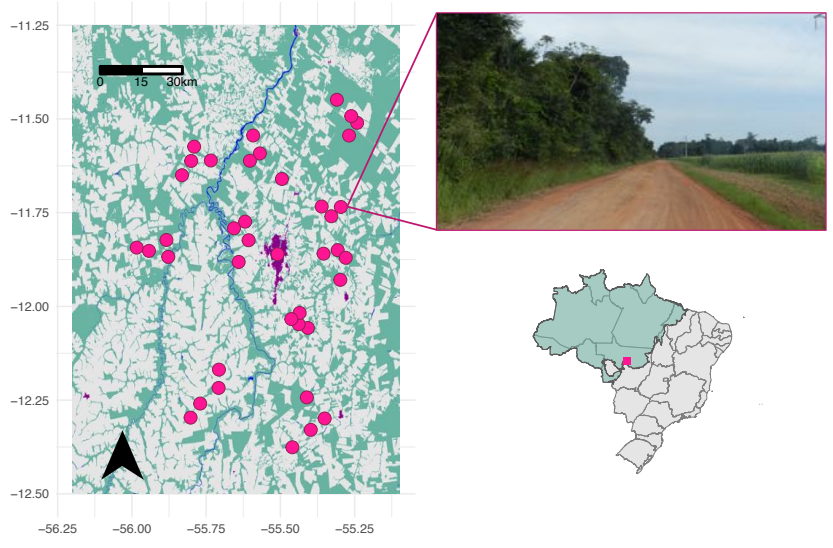

Figure 2: Species accumulation curves based on the species diversity found with either an iDNA sampler or with camera traps. Solid lines indicate interpolation while dashed lines indicate extrapolation. For the insect samples, extrapolation parameters were set to 1000 pooled samples to reflect the high number of total sandfly pooled samples. A pooled sample of insects is equal to either 50 sandflies, 15 mosquitos, or 5 carrion flies that were used for DNA extraction and metabarcoding. A camera day is equal to one 24-hour period for an individual camera. The shaded region surrounding each line shows the $95 \%$ confidence intervals.
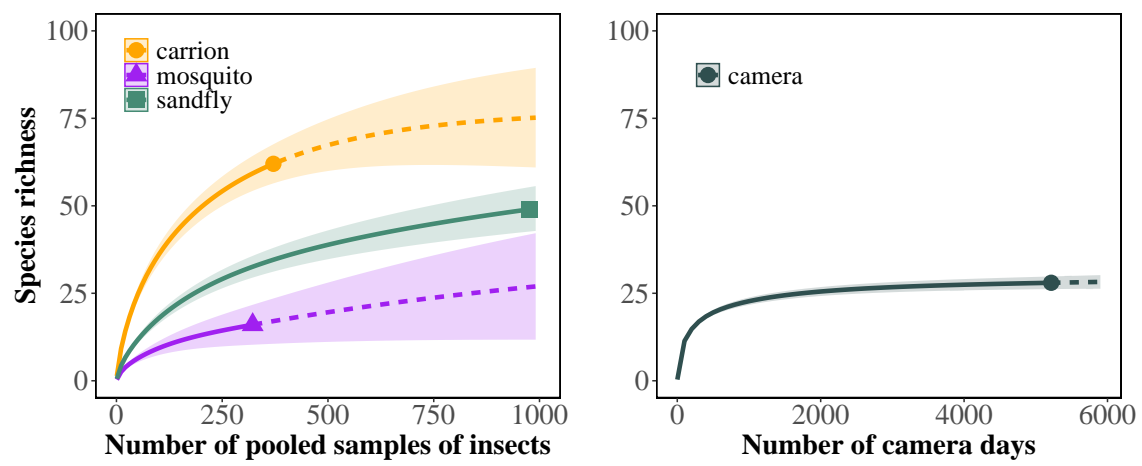

Figure 3: Relative abundance index (RAI) for each species present within either an iDNA sampler dataset 
or the camera trapping dataset. Species are divided into respective groups describing their taxa or a major life history trait to compare the effectiveness of each sampling method for describing vertebrate diversity. 


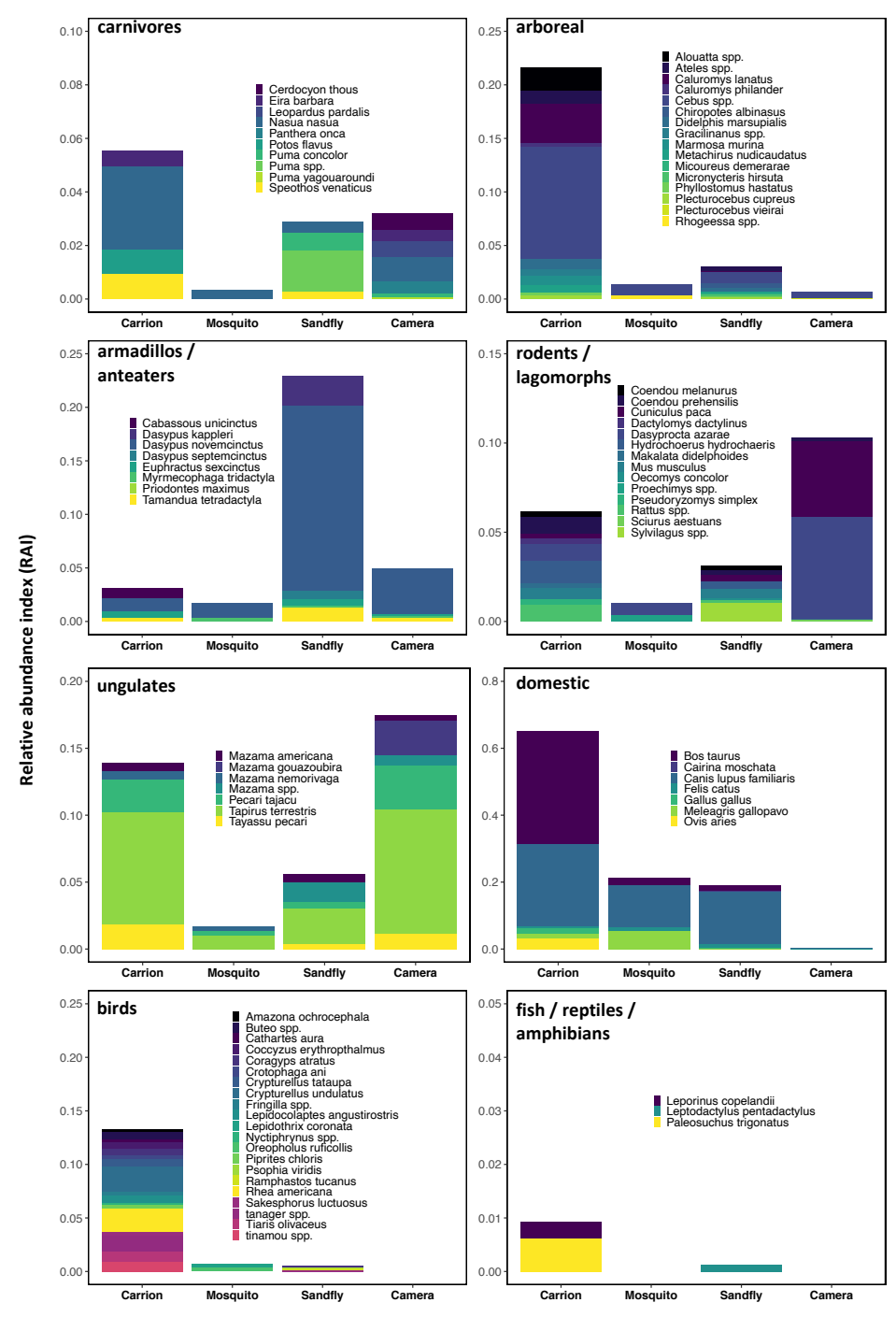


Figure 4: The median number of species found at each site for each sampling type. Boxplots are bounded by the first and third quantiles.

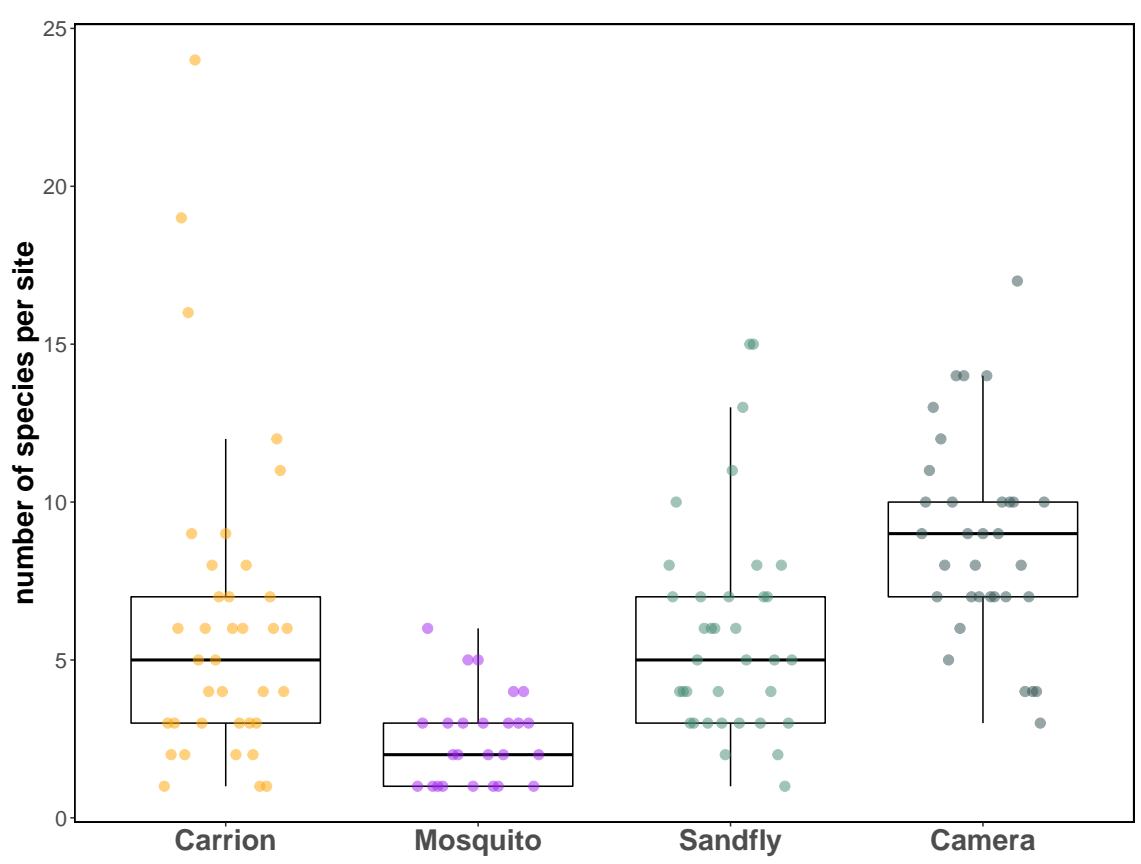

Figure 5: NMDS ordination showing overlap in vertebrate species communities as revealed by each iDNA source. Both panels display the same ordination with (A) showing the site labels colored by the iDNA sampler and $(B)$ showing significant species $(p$-value $<0.001)$ as vectors. 

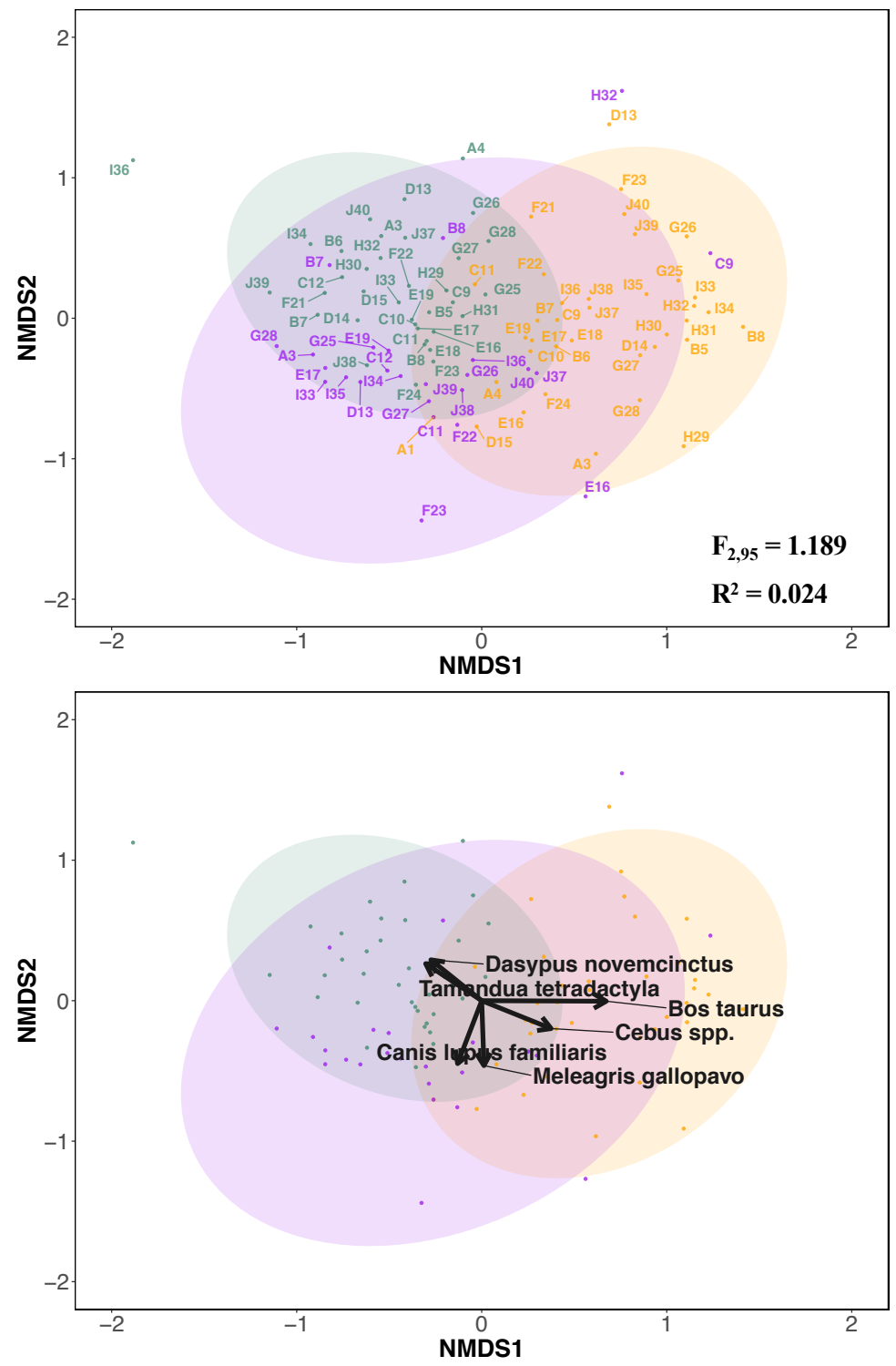

carrion

- mosq

- sandfly 
Figure 6: Proportion of dog, armadillo, cattle, and human positive samples measured at each site for each iDNA source. Data points show the proportion of total pooled samples at a site that were positive for the respective species.
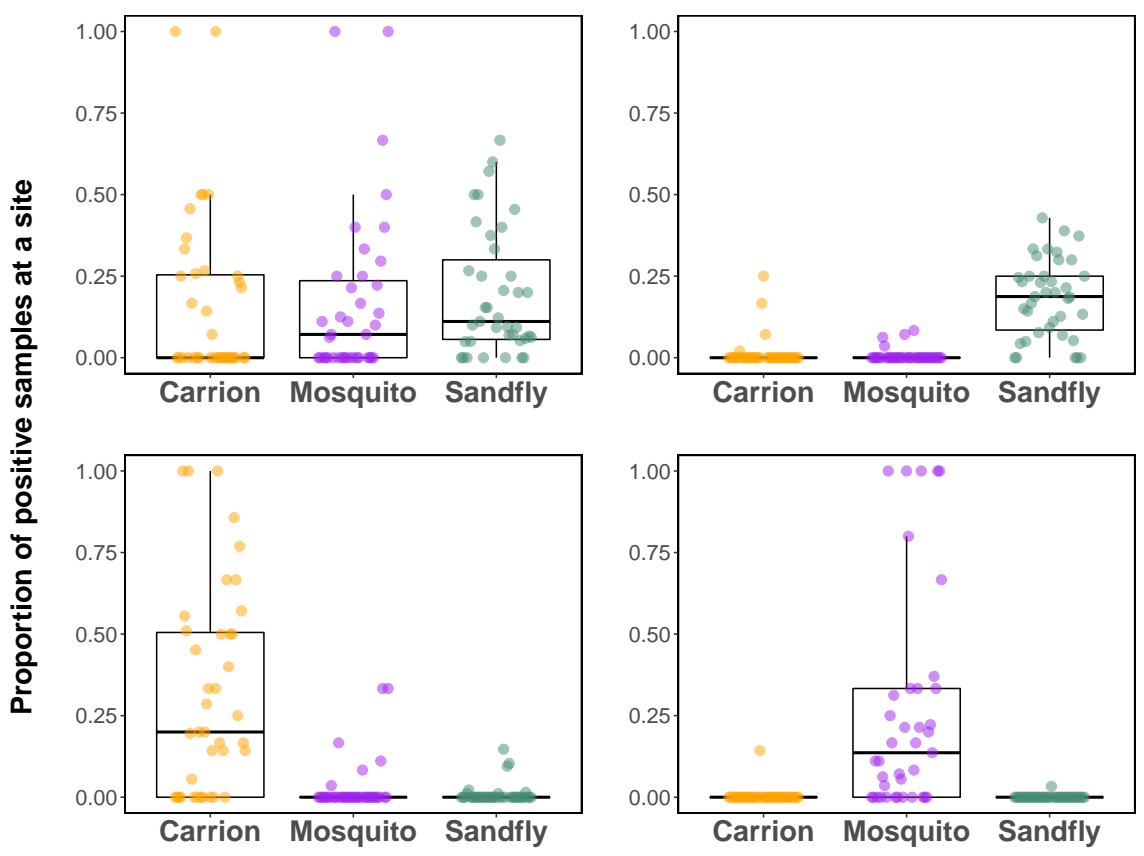